\title{
Brain metastasis PD-L1 and CD8 expression is dependent on primary tumor type and its PD-L1 and CD8 status
}

Florian Camy, ${ }^{1}$ Georgia Karpathiou (D) , ${ }^{1}$ Jean Marc Dumollard, ${ }^{1}$ Nicolas Magne, ${ }^{2}$ Jean Luc Perrot, ${ }^{3}$ Francois Vassal, ${ }^{4}$ Tiphanie Picot, ${ }^{1}$ Mousa Mobarki, ${ }^{1,5}$ Fabien Forest (D) , ${ }^{1}$ Francois Casteillo, ${ }^{1}$ Sirine Hathroubi, ${ }^{1}$ Marios Froudarakis, ${ }^{6}$ Michel Peoc' $h^{1}$
To cite: Camy F, Karpathiou G, Dumollard JM, et al. Brain metastasis PD-L1 and CD8 expression is dependent on primary tumor type and its PD-L1 and CD8 status. Journal for ImmunoTherapy of Cancer 2020;8:e000597. doi:10.1136/ jitc-2020-000597

- Additional material is published online only. To view, please visit the journal online (http://dx.doi.org/10.1136/jitc2020-000597).

FC and GK are joint first authors.

Accepted 23 June 2020

Check for updates

(C) Author(s) (or their employer(s)) 2020. Re-use permitted under CC BY-NC. No commercial re-use. See rights and permissions. Published by BMJ.

For numbered affiliations see end of article.

Correspondence to Dr Georgia Karpathiou; gakarpath@yahoo.gr

\section{ABSTRACT}

Background Brain metastases (Bmets) are frequent; however, limited data exist on the efficacy of immunotherapy in these lesions. The aims of the study were to analyze the immunohistochemical expressions of programmed death ligand 1 (PD-L1) and CD8 in Bmets and to compare them with their expressions in paired primary tumors, as well as correlate the results with clinicopathological features.

Methods This is a retrospective study of 233 patients with Bmets and 111 paired primaries. Clinical, histological, and molecular data were recorded and compared with the immunohistochemical results of PD-L1 and CD8 expressions. The statistical analysis included $\chi^{2}$ test, Cramer's V test, factorial analyses of variance, simple regression analysis, and Kaplan-Meier analysis with logrank product limit estimation.

Results PD-L1 expression was found in $23.6 \%$ of Bmets and in $29.0 \%$ of primary tumors with concordant expression between them in $75.5 \%$ of cases. Bmets PDL1 expression was associated with primary tumor PD-L1 expression and the primary tumor type. Significant CD8 peritumoral expression was found in $68.6 \%$ of Bmets and in $87.7 \%$ of primary tumors. CD8 expression was concordant between primary and metastatic tumors in $73.3 \%$ of cases. Bmets CD8 expression was associated with primary tumor CD8 expression and primary tumor type. PD-L1 expression was associated with CD8 expression in both primary and metastatic tumors. The concordance between primary and metastatic tumor PD-L1 expression was independent of all factors studied. The concordance between primary and metastatic CD8 expressions was marginally associated to the time of Bmets development. No prognostic role for PD$\mathrm{L} 1$ and CD8 expression in Bmets was found.

Conclusion PD-L1 and CD8 Bmets expressions are associated with the primary tumor type and its PD-L1 and CD8 expressions. No factor predicts the discordance for PD-L1 expression, while time to Bmets development is associated with CD8 expression discordance.

\section{INTRODUCTION}

Brain metastases (Bmets) are frequent, affecting from $10 \%$ to up to one-third of adult patients with cancer. ${ }^{12}$ They are the most common adult brain tumors, occurring 10 times more frequently than primary brain tumors. ${ }^{3}$ The most common cause of Bmets is pulmonary tumors, found in about $20 \%$ of the patients, followed by breast carcinoma and melanomas, with melanoma having the highest propensity to metastasize to the brain in comparison to other primaries. ${ }^{3}$ Other tumors showing a relatively high incidence for Bmets are gastrointestinal and renal tumors, ${ }^{34}$ while prostatic, thyroid, and ovarian cancers rarely cause them. ${ }^{3}$

Despite the fact that Bmets are frequent and are one of the most deleterious cancer localizations with important morbidity and mortality, many patients with Bmets are excluded from clinical trials, such that evidence of central nervous system (CNS) activity for the development of new drugs is rarely obtained. ${ }^{5}$ Immunotherapy, especially the blockade of inhibitory pathways which constrain the immune response, such as the 'immune-checkpoints' cytotoxic T-lymhocyteassociated protein 4 and programmed cell death 1 (PD-1), has gained great interest in the past few years due to impressive results in various tumor types. Anti-PD-1 and -programmed death ligand 1 (PD-L1) antibodies have been approved by the Food and Drug Administration for the treatment of melanoma, lung, renal, and head and neck cancers. ${ }^{4}$ Regarding the efficacy of immunotherapy in Bmets, relatively few data exist, ${ }^{6}$ as initial clinical trials had excluded these patients. The few and preliminary data show some intracranial responses in patients with melanoma or lung cancer Bmets treated by immunotherapy, especially in asymptomatic patients with no prior local treatment failure. ${ }^{4}$ 
The brain has long been considered as an immunoprivileged microenvironment; the influx of lymphocytes into the CNS is typically absent under normal circumstances, but disruption of the blood-brain barrier can occur in the presence of Bmets. The immune microenvironment under Bmets has been rarely explored. Similarly, the factors that could influence this microenvironment are unknown. Does a brain metastasis (BM) retain the same immunological characteristics as the primary tumor, such that the initial biopsy would be representative of the metastatic focus, or does it change according to the new microenvironment? Given the rarity of Bmets histological material, these questions are difficult to investigate.

The aim of this study was to analyze the immunohistochemical expression of two main immune factors-PD-L1, an inhibitory molecule used by tumor cells to avoid the host immune response, and CD8, a marker of cytotoxic $\mathrm{T}$ lymphocytes - in a large series of Bmets and to compare their expressions with that in paired primary tumors, in order to investigate whether the BM preserves the same profile as the parental tumor. Furthermore, we aimed to compare the BM immune microenvironment with the various clinical and pathological characteristics, in order to see if it is dependent on any of these factors, but also to investigate whether these factors have an impact on any possible discordance of the immune microenvironment between the BM and the primary tumor.

\section{MATERIALS AND METHODS \\ Study group}

This was a retrospective study of patients histologically diagnosed with Bmets between 1994 and 2017 at the University Hospital of Saint-Etienne. Clinical data regarding the $\mathrm{BM}$ and the primary tumor were retrieved from electronic files. The data included sex, age at BM diagnosis, primary tumor type, stage and treatment, time to BM development, BM location, multiplicity, size (further grouped in tumors of $\leq 30$ or $>30 \mathrm{~mm}$, as this is often considered as the cut-off for surgery ${ }^{1}$ ), symptomatology at BM presentation and performance status (PS), BM treatment, and survival after BM diagnosis. Survival was studied in association with clinical, histological, and immunohistochemical factors. All available histological and immunohistochemical slides from Bmets and primary tumors were examined in order to verify the correct diagnosis and to choose the most representative tissue for further analysis.

\section{Immunohistochemical evaluation}

An immunohistochemical study was performed in 233 Bmets and in 111 paired primary tumors. It was performed using $4 \mu \mathrm{m}$ thick full sections in an automated staining system (OMNIS, Dako-Agilent, Santa Clara, California, USA). Positive immunoreactions were visualized using $3,3^{\prime}$-diaminobenzidine as the chromogenic substrate. Primary antibodies included PD-L1 (Dako Agilent, 22C3, $1 / 50$, tonsil was used as positive control) and CD8 (Dako, C8/144B, 1/100).

PD-L1 expression (figure 1) was recorded as the percentage of tumor cells showing membranous staining; a cut-off value of $1 \%$ was used for further statistical analysis. ${ }^{6}$ Only tumor cell positivity was recorded. Cases were considered to have concordant PD-L1 expression when both tumor samples (primary and brain metastasis) were positive or negative, according to the cut-off value of $1 \%$. A semiquantitative system, as previously described, ${ }^{7-9}$ was applied to evaluate CD8 expression separately in the peritumoral and intratumoral compartments (figure 2): 0 : no cells $(0), 1$ : few cells $(<10 \%), 2$ : moderate number of positive cells $(\geq 10 \%$ and $<40 \%)$, and 3: abundant cells $(\geq 40 \%)$. A binary system of low (scores 0 and 1 ) and high (scores 2 and 3) CD8 lymphocytic response was used for further statistical analysis. For all analyses, the unanimous
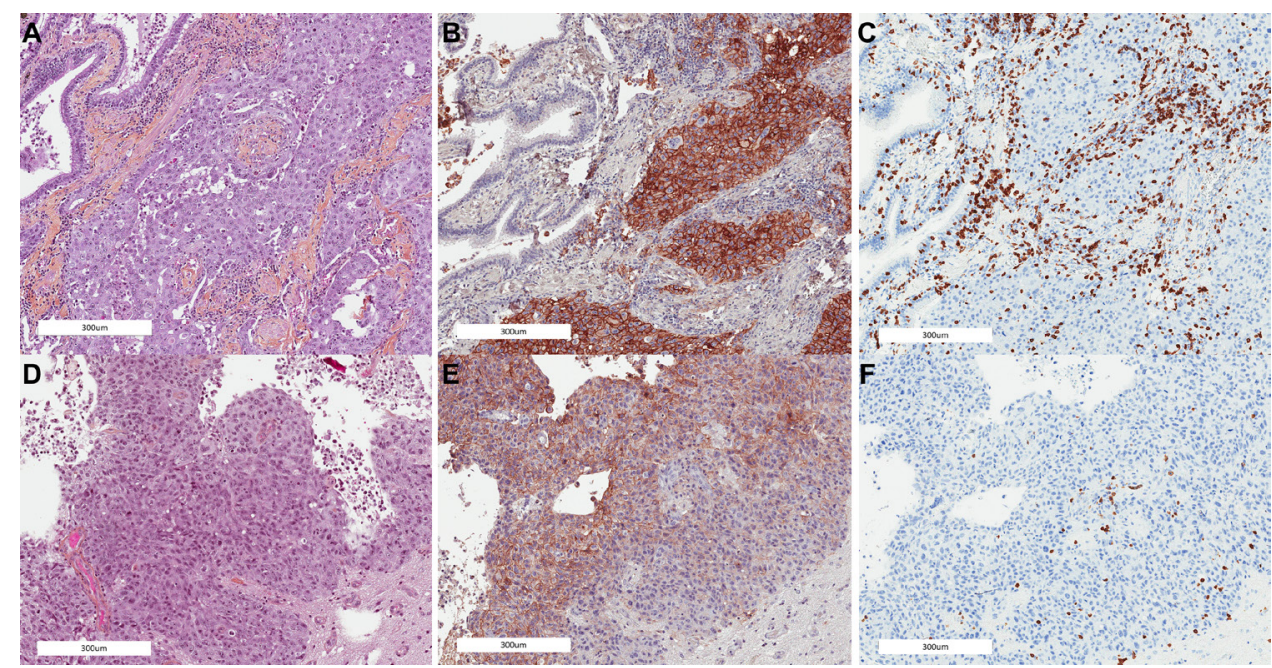

Figure 1 Pulmonary tumor compared with the corresponding brain metastasis showing concordant PD-L1 and CD8 expression: (A) primary tumor, (B) PD-L1 expression at the primary tumor, (C) CD8 expression at the primary tumor, (D) brain metastasis, (E) PD-L1 expression at the brain metastasis, and (F) CD8 expression at the brain metastasis. PD-L1, programmed death ligand 1. 


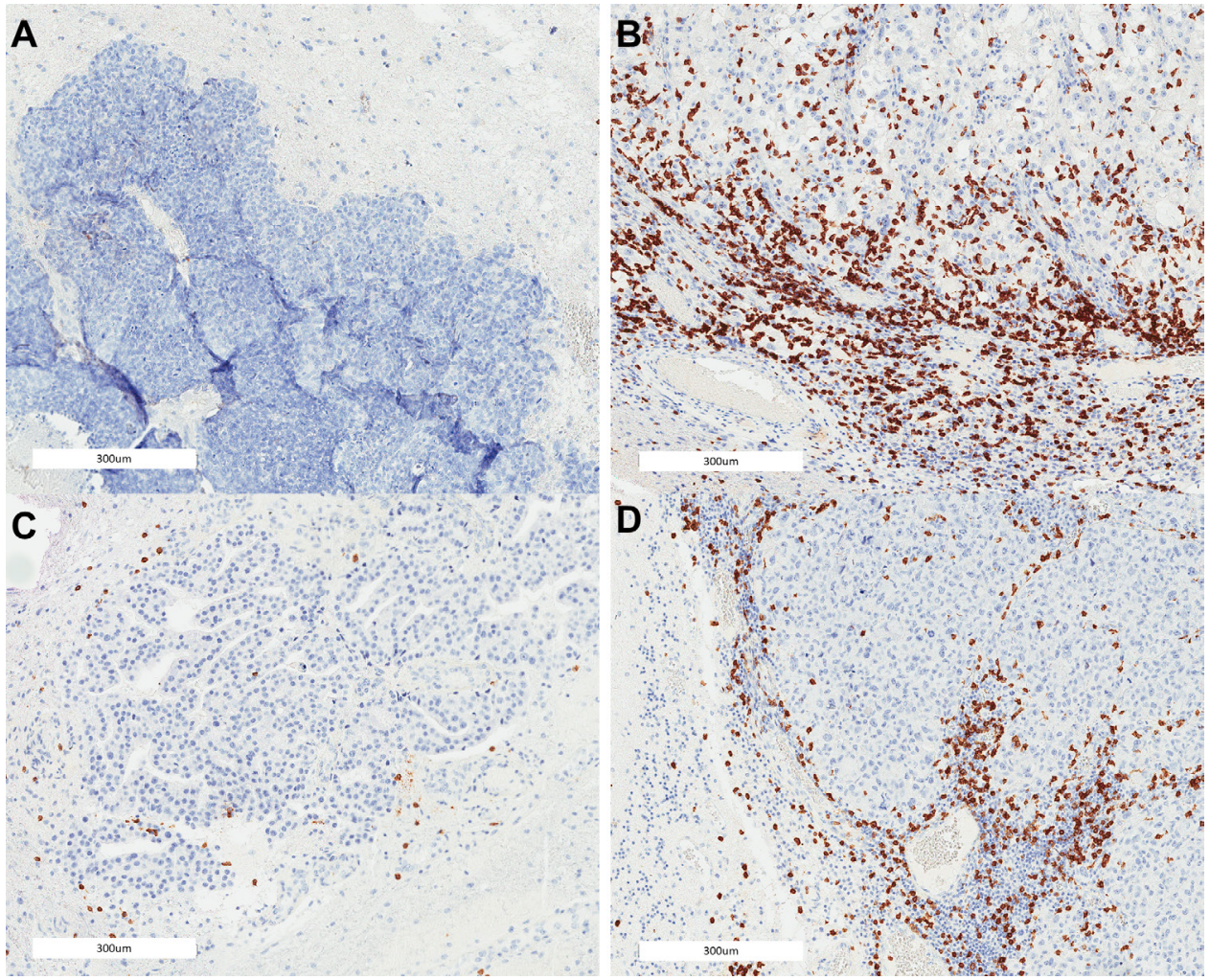

Figure 2 Illustration of CD8 lymphocytic scoring in brain metastases: (A) breast cancer brain metastasis without lymphocytic infiltration at the peritumoral or intratumoral compartment, $(B)$ renal cancer brain metastasis with severe lymphocytic infiltration at the peritumoral and intratumoral compartments, $(\mathrm{C})$ breast cancer brain metastasis with mild lymphocytic infiltration at the peritumoral and intratumoral compartments, and (D) melanoma brain metastasis with moderate lymphocytic infiltration at the peritumoral and intratumoral compartments.

estimation between pathologists (FC, GK, and JMD) was used as the final value.

\section{Molecular analysis}

Molecular analysis was performed for 93 cases (lung adenocarcinomas, melanomas, and gastrointestinal carcinomas), as previously described. ${ }^{10-12}$ Briefly, formalinfixed paraffin-embedded tumor sections were used for DNA extraction (automated extraction: Qiacube kit used; Qiagen, Courtaboeuf, France). DNA concentration and quality were assessed by the Qubit method and a Nanodrop spectrophotometer (Thermo Fisher Scientific, Waltham, Massachusetts, USA). Mutational analysis was performed by next-generation sequencing $(n=57)$ using the Ion Chef, the Torrent Personal Genome Machine, and the Colon and Lung Oncomine CE-IVD Panel (Thermo Fisher Scientific), as well as by SnaPshot ( $\mathrm{n}=36$; Applied Biosystems, Foster City, California, USA).

\section{Statistical analysis}

Data were analyzed using the StatView V.4.5 software (Abacus Concepts, Berkeley, California, USA). We used $\chi^{2}$ test to explore any relationship between two groups for categorical data, factorial analyses of variance (ANOVAs) to consider the effect of at least one factor on a continuous parameter studied, and simple regression analysis to explore a possible relationship between two continuous parameters. We also added Cramer's V, a post hoc test, to all $\chi^{2}$ analyses where more than $2 \times 2$ subgroups existed, in order to determine strengths of association after $\chi^{2}$ test determined significance. Cramer's value of $\geq 0.3$ denotes a strong association. Survival probability was estimated by Kaplan-Meier analysis with log-rank product limit estimation. For all analyses, statistical significance was indicated at a $\mathrm{p}$ value of $<0.05$.

\section{RESULTS \\ Patient and tumor characteristics}

The 233 patient cohort included 132 men (56.7\%) and 101 women $(43.3 \%)$ with an age range between 30 and 87 (median 61) years (table 1). Lung cancer was the most common cause of Bmets (34.8\%), followed by breast cancer $(13.3 \%)$, melanoma $(12.0 \%)$, and gastrointestinal $(12.0 \%)$ and renal $(7.7 \%)$ tumors; while there were fewer ovarian $(5.2 \%)$, head and neck $(3.4 \%)$, endometrial $(2.6 \%)$, thyroid $(2.6 \%)$, bladder $(2.6 \%)$, prostatic $(2.1 \%)$, and unknown primary $(1.7 \%)$ tumors. Most cases were surgically resected Bmets $(87.4 \%)$ and, in most of them $(92.2 \%)$, a complete resection was achieved. In $83.6 \%$ of cases, an adjuvant treatment was administrated. In $44.5 \%$ of patients, this involved radiotherapy only; $50.8 \%$ received chemotherapy and $7 \%$ hormonotherapy, while $1.7 \%$ received immunotherapy. For 111 cases, the material of the primary tumor was available and could 
Table 1 Patient and tumor characteristics

\section{n (\%)}

\begin{tabular}{lc}
\hline Sex $(n=233)$ & \\
Female & $101(43.3)$ \\
Male & \\
Age $(n=233)$ & $30-87$ \\
Range & $61,60.6( \pm 10.5)$ \\
Median, mean (SD) & \\
Metastasis localization $(n=215)$ & $66(30.7)$ \\
Frontal & $62(28.8)$ \\
Cerebellar & $29(13.5)$ \\
Temporal & $26(12.1)$ \\
Parietal & $26(12.1)$ \\
Occipital & $4(1.8)$ \\
Meningeal & $2(1)$ \\
Thalamic & \\
Multiple cerebral metastases $(n=216)$ & $56(25.9)$ \\
Yes & $160(74.1)$ \\
No &
\end{tabular}

Symptomatic metastasis with corticosteroid treatment $(n=205)$

$\begin{array}{cc}\text { Yes } & 185(90.2) \\ \text { No } & 20(9.8) \\ \text { Performance status }(n=206) & \\ 0-1 & 141(68.5) \\ 2-4 & 65(31.5)\end{array}$

Metastasis diagnosis in comparison to the primary tumor $(\mathrm{n}=228)$

\begin{tabular}{|c|c|}
\hline Revealing & $79(34.6)$ \\
\hline Metachronous & $149(65.4)$ \\
\hline \multicolumn{2}{|c|}{ Metastasis size (in $\mathrm{mm}, \mathrm{n}=113$ ) } \\
\hline Range & $6-70$ \\
\hline Median, mean (SD) & $30,31.9( \pm 13.4)$ \\
\hline \multicolumn{2}{|c|}{ Metastasis size (in $\mathrm{mm}, \mathrm{n}=113$ ) } \\
\hline$\geq 30$ & $53(46.9)$ \\
\hline$<30$ & $60(53.1)$ \\
\hline \multicolumn{2}{|c|}{ Primary tumor origin $(n=233)$} \\
\hline Lung & $81(34.8)$ \\
\hline Breast & $31(13.3)$ \\
\hline Gl tract & $28(12)$ \\
\hline Melanoma & $28(12)$ \\
\hline Kidney & $18(7.7)$ \\
\hline Ovary & $12(5.2)$ \\
\hline Head and neck & $8(3.4)$ \\
\hline Endometrium & $6(2.6)$ \\
\hline Thyroid gland & $6(2.6)$ \\
\hline Bladder & $6(2.6)$ \\
\hline Prostate & $5(2.1)$ \\
\hline Unknown & $4(1.7)$ \\
\hline
\end{tabular}

Continued
Table 1 Continued

$$
\text { n (\%) }
$$

Primary tumor histological type $(n=233)$

$\begin{array}{lc}\text { Adenocarcinoma } & 108(46.4) \\ \text { Undifferentiated carcinoma } & 37(10.3) \\ \text { Melanoma } & 28(12) \\ \text { Squamous cell carcinoma } & 24(10.3) \\ \text { Clear cell carcinoma } & 19(8.2) \\ \text { High-grade serous carcinoma } & 11(4.7) \\ \text { Large-cell neuroendocrine } & 4(1.7) \\ \text { carcinoma } & \\ \text { Urothelial carcinoma } & 2(0.9)\end{array}$

Cohort's molecular characteristics*

Lung tumors

$\begin{array}{lc}\text { EGFR mutated }(\mathrm{n}=35) & 2(5.7) \\ \text { KRAS mutated }(\mathrm{n}=28) & 10(36) \\ \text { ALK expression positive }(\mathrm{n}=20) & 0 \\ \text { BRAF mutated }(\mathrm{n}=18) & 0\end{array}$

Breast tumors $(n=27)$

$\begin{array}{ll}\text { Hormone receptors positive } & 13(46.4) \\ \text { HER2 positive } & 14(48.3)\end{array}$

Gl tumors $(\mathrm{n}=20)$

KRAS mutated

Melanoma $(\mathrm{n}=9)$

BRAF mutated

Type of primary tumor material ( $n=111)$

$\begin{array}{ll}\text { Biopsy } & 41(37) \\ \text { Resection } & 70(63)\end{array}$

T stage of primary tumor at initial diagnosis $(n=86)$

$$
\begin{array}{ll}
\text { Early (T1, T2) } & 43(50) \\
\text { Advanced (T3, T4) } & 43(50)
\end{array}
$$

$\mathrm{N}$ stage of primary tumor at initial diagnosis $(n=98)$

$\begin{array}{ll}\text { Negative } & 42(42.9) \\ \text { Positive } & 56(57.1)\end{array}$

$M$ stage of primary tumor at initial diagnosis $(n=103)$

$\begin{array}{ll}\text { Negative } & 62(60.2) \\ \text { Positive } & 41(39.8)\end{array}$

Metastasis material type $(\mathrm{n}=223)$

$\begin{array}{lr}\text { Biopsy } & \text { 28, (12.6) } \\ \text { Resection } & 195,(87.4)\end{array}$

Complete resection of brain metastasis $(n=192)$

$$
\begin{array}{lc}
\text { Yes } & 177(92.2) \\
\text { No } & 15(7.8)
\end{array}
$$

Adjuvant treatment after metastatectomy $(n=207)$

$$
\begin{array}{lr}
\text { Yes } & 173(83.6) \\
\text { No } & 34(16.4)
\end{array}
$$

Radiotherapy only as adjuvant treatment $(n=207)$ 
Table 1 Continued

\begin{tabular}{lc}
\hline & $\mathbf{n}(\%)$ \\
\hline Yes & $77(37.2)$ \\
No & $130(62.8)$ \\
$\begin{array}{l}\text { Median brain metastasis-free } \\
\text { survival (in months, } \mathrm{n}=111)\end{array}$ & 32 \\
$\begin{array}{l}\text { Median survival after brain } \\
\text { metastasis diagnosis (in months, } \\
\mathrm{n}=220)\end{array}$ & 13 \\
Status ( $\mathrm{n}=220)$ & \\
$\quad$ Dead & $174(79.1)$ \\
Alive & $46(20.9)$ \\
\hline
\end{tabular}

$\mathrm{n}$ denotes the number of cases from which the information was available.

${ }^{*} \mathrm{n}=$ cases where the molecular biology was performed; missing cases correspond to poor DNA quality or quantity. GI, gastrointestinal; HER2, human epidermal growth factor receptor 2.

be studied. The median period between the diagnosis of the primary tumor and the appearance of Bmets was 32 months, and the median overall survival after Bmet diagnosis was 13 months (online supplementary figure S1).

\section{Immunohistochemical analysis and correlation with clinical and histological factors}

PD-L1 expression was found in $23.6 \%$ of Bmets and in $29.0 \%$ of primary tumors with a concordant expression (cut-off of $1 \%$ ) between them in $75.5 \%$ of cases (table 2). More specifically, 12 cases (11.3\%) showed primary but not metastatic tumor PD-L1 expression; 14 cases (13.2\%) showed metastatic but not primary tumor PD-L1 expression; 19 cases (18\%) showed PD-L1 expression in both the primary tumor and the BM; and, finally, 61 cases $(57.5 \%)$ did not show PD-L1 expression in neither primary tumor nor BM (online supplementary figure S2). PD-L1 positivity of the primary tumor (counted as percentage) was directly associated with the positivity of the metastatic focus in a simple regression analysis $(\mathrm{p}=0.0002$, online supplementary figure S3). More specifically, 59.6\% of patients showed the same percentage of tumor cell expression between the two tumor compartments, while $18.3 \%$ showed higher expression in the Bmets and 22.1\% showed lower expression in the Bmets. Significant CD8 peritumoral expression was found in $68.6 \%$ of Bmets and in $87.7 \%$ of primary tumors, while intratumoral CD8 expression was found in $43.8 \%$ of Bmets and in $61.5 \%$ of primary tumors. CD8 expression was concordant between primary and metastatic tumors in $73.3 \%$ and $47.8 \%$ of cases for peritumoral and intratumoral expression, respectively. Bmets CD8 lymphocytic response was directly associated with primary tumor CD8 response $(\mathrm{p}=0.01)$. When seen by percentage, CD8 expression was similar between primary and metastatic tumors in $41 \%$ of cases, lower in the metastatic tumor in $47.6 \%$ of cases, and higher in the metastatic tumor in $11.4 \%$ of cases.
Table 2 Immunohistochemical study

\begin{tabular}{lr} 
Variable & $\mathbf{n}(\%)$ \\
\hline PD-L1 expression by the metastasis $(\mathrm{n}=229)$ & $54(23.6 \%)$ \\
Yes & $175(76.4 \%)$ \\
No
\end{tabular}

PD-L1 expression by the metastasis for positive cases $(n=54)$

\begin{tabular}{lr} 
Range & \multicolumn{1}{c}{$1-100$} \\
Median, mean (SD) & $3,12.3 \pm 22.4$ \\
Peritumoral CD8 expression by the metastasis $(\mathrm{n}=223)$ & $70(31.4 \%)$ \\
No/mild & $153(68.6 \%)$ \\
Moderate/severe & $123(56.2 \%)$ \\
Intratumoral CD8 expression by the metastasis $(\mathrm{n}=219)$ \\
No/mild & $96(43.8 \%)$ \\
Moderate/severe & $31(29 \%)$ \\
PD-L1 expression by the primary tumor $(\mathrm{n}=107)$ \\
Yes \\
No
\end{tabular}

PD-L1 expression by the primary tumor for positive cases $(\mathrm{n}=31)$

\begin{tabular}{|c|c|}
\hline Range & $1-100$ \\
\hline Median, mean (SD) & $17.5,26.5 \pm 30.1$ \\
\hline \multicolumn{2}{|c|}{ Peritumoral CD8 expression by the primary tumor $(n=106)$} \\
\hline No/mild & $13(12.3 \%)$ \\
\hline Moderate/severe & $93(87.7 \%)$ \\
\hline \multicolumn{2}{|c|}{ Intratumoral CD8 expression by the primary tumor $(n=96)$} \\
\hline No/mild & $37(38.5 \%)$ \\
\hline Moderate/severe & $59(61.5 \%)$ \\
\hline \multicolumn{2}{|c|}{$\begin{array}{l}\text { Concordant PD-L1 expression between paired primary } \\
\text { tumor and metastases }(n=106)\end{array}$} \\
\hline Yes & $80(75.5 \%)$ \\
\hline No & $26(24.5 \%)$ \\
\hline
\end{tabular}

Concordant peritumoral CD8 expression between paired primary tumor and metastases $(n=101)$

$\begin{array}{ll}\text { Yes } & 74(73.3 \%) \\ \text { No } & 27(26.7 \%)\end{array}$

Concordant intratumoral CD8 expression between paired primary tumor and metastases $(n=92)$

$\begin{array}{ll}\text { Yes } & 44(47.8 \%) \\ \text { No } & 48(52.2 \%)\end{array}$

$\mathrm{n}$ denotes the number of cases from which the information was available.

PD-L1, programmed death ligand 1.

PD-L1 expression was associated with CD8 expression in both primary $(\mathrm{p}=0.0003$ for intratumoral and 0.07 for peritumoral CD8 expression) and metastatic $(\mathrm{p}<0.0001$ for intratumoral and peritumoral expression) tumors, as most PD-L1 expressing tumors were those with intense lymphocytic response (table 3 ). 
Table 3 Correlation between PD-L1 and CD8 expression

PD-L1 brain metastasis expression

No Yes P value

\begin{tabular}{cccc}
\hline CD8 peritumoral brain metastasis expression & \\
Low & 65 & 4 & $<0.0001$ \\
High & 104 & 49 &
\end{tabular}

CD8 intratumoral brain metastasis expression

\begin{tabular}{cccc} 
Low & 110 & 13 & $<0.0001$ \\
\hline High & 55 & 40 & \\
PD-L1 primary & tumor expression & \\
\hline Yes & 12 & 19 & $<0.0001$ \\
No & 61 & 14 & \\
\multicolumn{5}{c}{ PD-L1 primary tumor expression } \\
CD8 peritumoral primary tumor expression & P value \\
\hline Low & 12 & 1 & 0.0719 \\
\hline High & 62 & 29 & \\
CD8 intratumoral primary tumor expression \\
\hline Low & 34 & 3 & 0.0003 \\
\hline High & 34 & 25 & \\
\hline
\end{tabular}

PD-L1, programmed death ligand 1.

The concordance between primary and metastatic tumor PD-L1 expressions was independent of the tumor origin $(\mathrm{p}=0.4)$ and of the time to metastasis development for all tumors $(\mathrm{p}=0.5)$ and separately for pulmonary $(\mathrm{p}=0.8)$, breast $(\mathrm{p}=0.8)$, gastrointestinal $(\mathrm{p}=0.2)$, and renal $(\mathrm{p}=0.2)$ tumors, as well as melanomas $(\mathrm{p}=0.9)$. It was also independent of sex $(\mathrm{p}=0.1)$, age $(\mathrm{p}=0.8)$, type of material $(p=0.7)$, metastasis location $(p=0.7)$, metastases multiplicity $(\mathrm{p}=0.6)$, of $\mathrm{BM}$ revealing (synchronous) the primary tumor $(\mathrm{p}=0.3)$, BM size $(\mathrm{p}=0.2)$, corticosteroid treatment $(\mathrm{p}=0.7)$, the $\mathrm{T}(\mathrm{p}=0.7)$ and $\mathrm{N}(\mathrm{p}=0.07)$ stages of the primary tumor, and its histology $(\mathrm{p}=0.3)$.

The concordance between primary and metastatic peritumoral CD8 expressions was independent of the tumor origin $(\mathrm{p}=0.08)$. It was marginally associated with the time of BM development, as BM with discordant CD8 expression presented 7 months later ( $\mathrm{p}=0.05$, online supplementary figure S1). Regarding concordant intratumoral CD8 expression, there was no association with the time to metastasis for all tumors $(\mathrm{p}=0.9)$ or separately for different tumor types. Concordance for CD8 expression was independent of age $(\mathrm{p}=0.4)$, sex $(\mathrm{p}=0.9)$, type of material $(\mathrm{p}=0.4)$, metastasis location $(\mathrm{p}=0.1)$, metastases multiplicity $(\mathrm{p}=0.2)$, of metastases revealing the primary tumor $(\mathrm{p}=0.3)$, corticosteroid treatment $(\mathrm{p}=0.9)$, BM size $(\mathrm{p}=0.2)$, and the $\mathrm{T}(\mathrm{p}=0.7)$ and $\mathrm{N}(\mathrm{p}=0.6)$ stages of the primary tumor.

BM PD-L1 expression (online supplementary table S1) was associated with the primary tumor origin $(\mathrm{p}=0.04)$, with pulmonary, renal, and ovarian tumors, as well as melanomas being the most often positive tumors, while it was independent of other characteristics such as age $(\mathrm{p}=0.9)$, sex $(p=0.2)$, BM location $(p=0.4)$, multiplicity $(p=0.5)$ or size $(\mathrm{p}=0.4)$, as well as the time to metastasis development $(\mathrm{p}=0.3)$, PS $(\mathrm{p}=0.1)$, or initial tumour, node, metastasis (TNM) stage. BM PD-L1 expression was independent of the material (biopsy or resection) used $(p=0.1)$. It was also independent of the corticosteroid treatment $(\mathrm{p}=0.1)$. For pulmonary primaries, PD-L1 Bmets positivity was not associated with EGFR mutation ( $\mathrm{p}=0.4)$ or KRAS mutation $(\mathrm{p}=0.1)$. For mammary tumors, it was not associated with hormone receptor positivity $(\mathrm{p}=0.3)$ but was associated with HER2 overexpression, as PD-L1 positive Bmets were associated with HER2 negativity $(\mathrm{p}=0.03)$. For gastrointestinal tumors, PD-L1 Bmets expression was not associated with KRAS mutation ( $\mathrm{p}=0.2)$. For melanomas, PD-L1 Bmets expression was not associated with $B R A F$ mutation $(\mathrm{p}=0.1)$.

CD8 Bmets expression was associated with the primary tumor origin ( $\mathrm{p}=0.0002$ for peritumoral expression and 0.008 for intratumoral) and histology $(\mathrm{p}=0.007)$, with lung and renal tumors, melanoma, and squamous cell carcinoma being mostly infiltrated by CD8+ lymphocytes. Peritumoral CD8 expression at the metastatic focus was associated with the type of material, as it was more often found in resection specimens $(p=0.004)$. CD8 lymphocytic response in BM was not associated with any other factor, including the corticosteroid treatment $(\mathrm{p}=0.2)$. For pulmonary primaries, CD8 Bmets positivity was not associated with EGFR mutation $(\mathrm{p}=0.3)$ or KRAS mutation $(\mathrm{p}=0.2)$. For mammary tumors, CD8 Bmets expression was not associated with hormone receptor positivity $(\mathrm{p}=0.09)$ or HER2 overexpression $(\mathrm{p}=0.4)$. For gastrointestinal tumors, CD8 Bmets expression was not associated with KRAS mutation $(\mathrm{p}=0.1)$. For melanomas, CD8 Bmets expression was not associated with $B R A F$ mutation $(\mathrm{p}=0.4)$.

Primary tumor PD-L1 expression was not associated with any of the factors studied (online supplementary table S2). CD8 expression at the primary tumor was associated with the BM location, with parietal tumors arising from primaries heavily infiltrated by CD8 lymphocytes, while occipital BM from primaries were less infiltrated by CD8 cells $(p=0.02)$. Furthermore, richer CD8 infiltration at the primary tumor was associated with single $\mathrm{BM}(\mathrm{p}=0.04)$. Primary tumors revealed by the BM (revealing metastases) were less infiltrated by CD8 cells $(\mathrm{p}=0.02)$. Furthermore, it was marginally associated $(\mathrm{p}=0.05)$ with $\mathrm{N}$ stage and sex.

When analyzing the various clinical and histological factors, a correlation was found between BM location and the primary tumor's origin ( $\mathrm{p}=0.0003)$, as lung primaries affected all locations almost equally; gastrointestinal, ovarian, and breast tumors led mostly to cerebellar metastases, while melanoma predominantly led to frontal Bmets. However, it is worth reiterating that the cases studied are only cases that have been operated on; thus, these results should be evaluated with caution.

\section{Survival analysis}

Patient survival after BM diagnosis was associated (online supplementary figure S4) with the PS $(\mathrm{p}<0.0001)$, the age of the patient $(\mathrm{p}=0.03)$, and the administration of adjuvant 
therapy ( $\mathrm{p}=0.002)$. It was not associated with the different types of adjuvant treatments. It was also correlated with the primary tumor's origin $(\mathrm{p}=0.03)$ and marginally $(\mathrm{p}=0.05)$ with the tumor histology. It was not associated with the rest of the factors studied (table 4 and figure 3 ).

A survival analysis separately performed for the different tumor types showed that, for lung cancer BM, better prognosis $(\mathrm{p}=0.02)$ was seen with adenocarcinoma (20 months' median survival), as compared with squamous cell carcinoma (13 months) or undifferentiated carcinoma (9 months); BM smaller size $(\mathrm{p}=0.02)$ and BM location $(\mathrm{p}=0.006)$ in patients with cerebellar metastases showed a median survival of 8 months, with frontal, temporal, parietal, and occipital metastases yielding survivals of $13,16,18$, and 20 months, respectively. CD8 BM intratumoral expression was marginally associated with better prognosis $(\mathrm{p}=0.07)$ for lung cancer $\mathrm{BM}$. The possibility of resection of the BM also retained its prognostic significance for different tumor types (lung tumors $\mathrm{p}=0.009,5$ vs 14 months; melanoma $\mathrm{p}=0.04,7$ vs 13 months; and renal $\mathrm{p}=0.003,3$ vs 23 months). No differences were noted for the rest of the factors studied.

Bmets-free survival after the diagnosis of the primary tumor (online supplementary table S3) was associated with the primary tumor origin $(\mathrm{p}<0.0001)$, where breast carcinomas and melanomas gave the latest metastases; the sex, reflecting breast tumors (multiple ANOVAs); and, as earlier mentioned, with the concordance of CD8 expression. It was not associated with other factor studies. No further correlations were found when analyzing by tumor groups.

\section{DISCUSSION}

We found that the brain metastasis PD-L1 expression is dependent on the primary tumor's characteristicsnamely, the type of the primary tumor and its PD-L1 status-while being independent of all other metastasis characteristics, such as multiplicity, size, location, and time to metastasis, or other primary tumor characteristics such as initial TNM stage or patient age, sex, and PS. This has important clinical consequences, as the primary tumor's PD-L1 status can be predictive of the corresponding brain metastasis PD-L1 status with no need for further histological material in $75 \%$ of cases. The discordance in $25 \%$ of the cases was not associated with any tumor or patient characteristics; thus, it seems to be an intrinsic feature of some tumors, with no factors predictive of this discordance. A similar percentage of tumors showed concordant peritumoral CD8 expression. Discordant cases were seen when Bmets were sampled later than the concordant cases, while no other factors were associated with discordance in CD8 expression. Bmets CD8 expression was dependent on primary tumor type and its CD8 infiltration, while being independent of other characteristics. Furthermore, our results of almost 25\% BM PD-L1 expression and 70\% BM CD8 expression highlight that an important part of Bmets could be amenable to immunomodulatory treatments. Among the factors proposed to predict the sensitivity to immunotherapy (ie, the "cancer immunogram') are the infiltration of the tumor by $\mathrm{T}$ cells and the presence of immunotherapy targets such as PD-L1 $1{ }^{13}$ which, as we show here, are indeed present in an important part of Bmets. Few previous studies exist which have investigated the immune microenvironment of Bmets; they were in line with our results, showing an overall PD-L1 expression in $30 \%$ of Bmets. ${ }^{7}$ Separately for breast cancer ${ }^{14}$ and melanoma Bmets, ${ }^{8}$ the expression reached $50 \%$ in the cases.

Interestingly, we showed that higher CD8 expression was associated with higher PD-L1 expression, not only for the primary but also for the metastatic focus, showing that, even in the brain metastasis micro-environment, an important lymphocytic reaction still exists, which
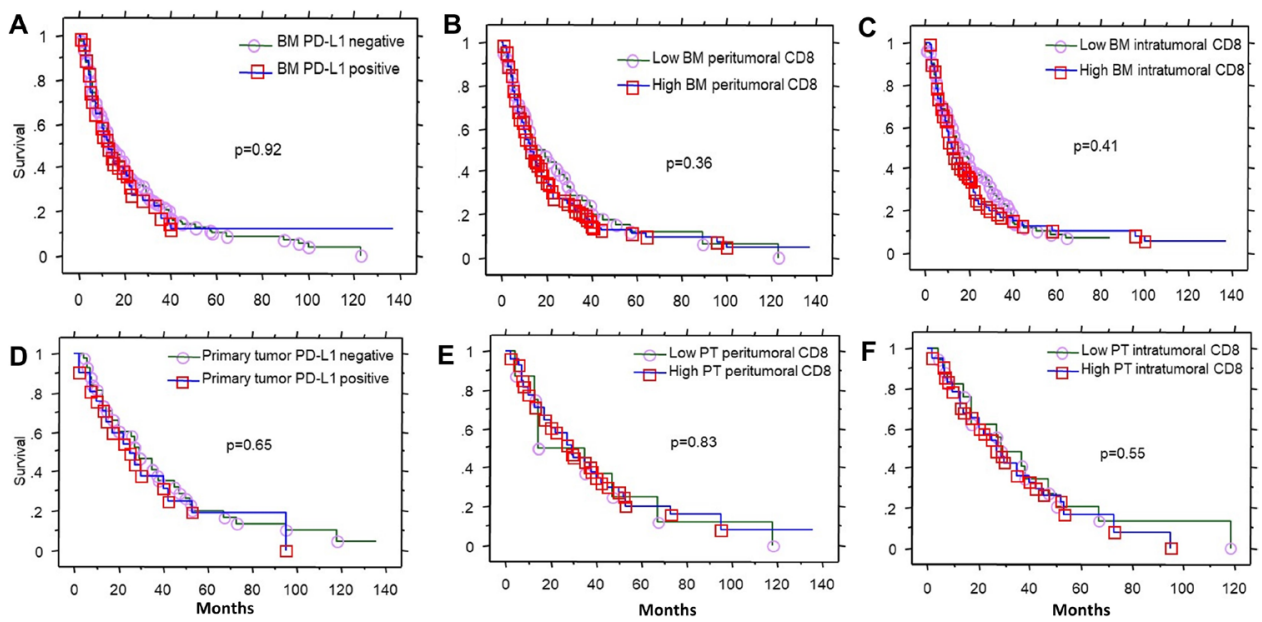

Figure 3 Overall survival after brain metastasis diagnosis according to (A) PD-L1 BM expression, (B) CD8 peritumoral BM expression, and (C) CD8 intratumoral BM expression. Overall survival after primary tumor diagnosis, according to (D) PT PD-L1 expression, (E) PT CD8 peritumoral expression, and (F) PT CD8 intratumoral expression. Low CD8=groups 0 and 1 ( $<10 \%$ CD8 expression), high CD8 expression=groups 2 and 3 ( $\geq 40 \%$ CD8 expression), PD-L1 positive: $\geq 1 \%$. BM, brain metastasis; PD-L1, programmed death ligand 1; PT, primary tumor. 
Table 4 Analysis of survival after brain metastasis diagnosis

\begin{tabular}{llc}
\hline & $\begin{array}{l}\text { Survival } \\
\text { (months) }\end{array}$ & P value \\
\hline PD-L1 brain-metastasis expression & & \\
Yes & 20 & 0.9 \\
No & 19 & \\
\hline
\end{tabular}

CD8 peritumoral brain metastasis expression

$\begin{array}{lll}\text { Low } & 21 & 0.3 \\ \text { High } & 18 & \end{array}$

CD8 intratumoral brain metastasis expression

$\begin{array}{lcc}\text { Low } & 19 & 0.4 \\ \text { High } & 18 & \end{array}$

CD8 brain metastasis and primary tumor expression association
Similarly high in the two
12
0.7 compartments

Similarly low in the two $\quad 14$

compartments

Higher in the brain metastasis than 21

the primary tumor

Lower in the brain metastasis than 19 the primary tumor

Primary tumor origin

$\begin{array}{lrr}\text { Lung } & 14 & 0.03 \\ \text { Breast } & 29 & \\ \text { Melanoma } & 7 & \\ \text { Gl } & 11 \\ \text { Renal } & 22 \\ \text { Ovary } & 13 \\ \text { Head neck } & 13\end{array}$

Age at brain metastasis diagnosis (years)

$\begin{array}{lll}>60 & 12 & 0.03 \\ \leq 60 & 18 & \end{array}$

Sex

$\begin{array}{lcc}\text { Female } & 19 & 0.05 \\ \text { Male } & 13 & \end{array}$

Histological type

$\begin{array}{lrr}\text { Adenocarcinoma } & 19 & 0.05 \\ \text { Squamous cell carcinoma } & 13 & \\ \text { Clear cell carcinoma } & 22 \\ \text { Melanoma } & 7 \\ \text { Undifferentiated } & 11 \\ \text { Large-cell neuroendocrine carcinoma } & 15 \\ \text { High-grade serous carcinoma } & 10 \\ \text { Urothelial carcinoma } & 23\end{array}$

Cohort's molecular characteristics

\begin{tabular}{llc} 
Lung tumors & & \\
EGFR mutated & 33 & 0.3 \\
EGFR non mutated & 21 & \\
\hline & & Continued
\end{tabular}

Table 4 Continued

\begin{tabular}{lcc} 
& $\begin{array}{l}\text { Survival } \\
\text { (months) }\end{array}$ & P value \\
\hline KRAS mutated & 25 & 0.7 \\
KRAS non mutated & 21 & \\
Breast tumors & & \\
Hormone receptors positive & 26 & 0.4 \\
Hormone receptors negative & 25 & \\
HER2 positive & 34 & 0.03 \\
HER2 negative & 20 & \\
GI tumors & & \\
KRAS mutated & 11 & 0.1 \\
KRAS non mutated & 13 & \\
Melanoma & & 0.08 \\
BRAF mutated & 22 & \\
BRAF non mutated & 6 &
\end{tabular}

Performance status

$\begin{array}{rrr}0-1 & 21 & <0.0001 \\ 2-4 & 5 & \end{array}$

Symptomatic Bmets requiring corticosteroid treatment

$\begin{array}{lll}\text { Yes } & 13 & 0.4 \\ \text { No } & 16 & \end{array}$

Metastases revealing the primary tumor

$\begin{array}{lrr}\text { Yes } & 12 & 0.7 \\ \text { No } & 13 & \end{array}$

No

13

Multiples Bmets

$\begin{array}{lll}\text { Yes } & 10 & 0.2 \\ \text { No } & 14 & \end{array}$

Size of brain metastasis $(\mathrm{mm})$

$\begin{array}{lll}\leq 30 & 12 & 0.5 \\ >30 & 11 & \end{array}$

\section{Bmets location}

$\begin{array}{lll}\text { Frontal } & 13 & 0.3 \\ \text { Cerebellar } & 11 & \\ \text { Occipital } & 21 & \\ \text { Parietal } & 16 & \\ \text { Temporal } & 13 & \\ \text { stage of the primary tumor } & & 0.4 \\ \text { 1-2 } & 20 & \\ 3-4 & 11 & \\ \text { N stage of the primary tumor } & & \end{array}$

$\mathrm{N}$ stage of the primary tumor

$\begin{array}{lcc}\mathrm{N}- & 13 & 0.6 \\ \mathrm{~N}+ & 17 & \\ \mathrm{M} \text { stage of the primary tumor } & & \\ \text { M0 } & 17 & 0.2 \\ \text { M1 } & 12 & \end{array}$

Adjuvant treatment after metastatectomy

\begin{tabular}{ccc} 
Yes & 17 & 0.002 \\
\hline & Continued
\end{tabular}


Table 4 Continued

\begin{tabular}{ccc}
\hline & $\begin{array}{l}\text { Survival } \\
\text { (months) }\end{array}$ & P value \\
\hline No & 5 & \\
Resection of the primary tumor & & \\
Yes & 16 & 0.4 \\
No & 9 & \\
Complete resection of brain metastasis & & \\
Yes & 14 & 0.8 \\
No & 13 & \\
\hline
\end{tabular}

Bmets, brain metastases; GI, gastrointestinal; HER2, human epidermal growth factor receptor 2; PD-L1, programmed death ligand 1.

provokes tumor cells to express the PD-L1 factor. In contrast, a previous study did not show any association between PD-L1 expression and lymphocytic infiltration in Bmets. ${ }^{7}$ As for the types of Bmets that provoke the most intense lymphocytic response, Harter et $a{ }^{15}$ in a study using tissue microarrays-a technique that could pose some difficulties due to intratumoral heterogeneityshowed that infiltration by immune cells was higher for renal cell carcinoma $\mathrm{BM}$, followed by melanoma BM, while breast and lung tumor BM showed poor immune response; another study ${ }^{7}$ showed higher infiltration for melanoma BM, followed by renal and lung cancer BM. Our results confirm the results of the latter study.

Regarding the comparison of the immune status between paired primary tumors and Bmets, very few studies exist. In a study of 74 lung cancer cases, ${ }^{16}$ there was a concordance in $86 \%$ of cases for PD-L1 expression; the discordance found in 10 cases was not statistically correlated with any particular factor, such as the time between the diagnosis of the primary and the metastatic tumor, even if most paired lesions with discordant PD-L1 expression were seen with specimens obtained six or more months apart. On the contrary, a study ${ }^{17}$ of 25 patients with paired primary and BM lung cancers found a $72 \%$ concordance in PD-L1 expression; discordance was mostly seen with synchronous rather than metachronous lesions. This is rather surprising and was not confirmed by the current series; it could be due to the limited number of cases in the aforementioned study. Interestingly, Goldberg et al treated lung cancer and melanoma brain metastatic patients with pembrolizumab. ${ }^{6}$ Their study included only patients with small asymptomatic metastases, such that its safety and efficacy in larger or symptomatic Bmets remain unknown. ${ }^{6}$ They found a high concordance between systemic and brain metastasis responses, which were also durable. Our results were consistent with these findings, showing a similar immunohistochemical profile between primary and metastatic foci regarding PD-L1 and CD8 (in most cases). We also showed that metastasis size, symptomatology, and PS were not associated with BM PD-L1 expression, nor did CD8 infiltration.
Regarding the prognostic role of PD-L1 and CD8 expressions in BM, we did not find any statistically significant association with survival in the whole group, nor in the separate analysis of different tumor groups. We did find, however, a marginal association of CD8 intratumoral BM expression with better survival in lung cancer BM. No prognostic significance was found by Harter et $a l,{ }^{15}$ while another group ${ }^{7}$ showed that CD8+ lymphocytes in BM were a favorable prognostic factor. Among all other factors studied, the PS, the age at BM diagnosis, the primary tumor type, and the use of treatment additional to the surgery were important prognostic factors. Separately for lung cancer Bmets, brain metastasis size, its location, and the possibility of resection of the BM were also prognostic factors. Furthermore, for this tumor group, histological type was significantly associated with survival, as patients diagnosed with adenocarcinomas had better survival after BM diagnosis. In a previous study, we showed that even grading of lung adenocarcinoma Bmets has an important prognostic impact. ${ }^{12}$

Furthermore, we found important correlations between the primary tumor's infiltration by CD8-positive lymphocytes and brain metastasis characteristics. Specifically, primary tumors less infiltrated by CD8 cells gave multiple BM most often, as well as metastases that reveal the primary tumor and occipital metastases. However, the number of cases was limited for this latter correlation, so the results should be interpreted with caution.

Our study has certain limitations. First, it is a retrospective study. Another limitation when analyzing the immune microenvironment in brain tumors is whether prior corticosteroid treatment can affect its elements. Interestingly, it seems that corticosteroids do not affect the amount of lymphocyte or PD-L1 expression, as studied in melanoma Bmets $^{78}$ and as we have shown in the current series. Another issue is the different clones and methods for PD-L1 immunohistochemical analysis; however, the clone that we used is one of the most commonly used in the various related studies. Furthermore, different cut-off values for PD-L1 have been used in the literature, but a cut-off of $1 \%$ (as presently used) is one of the most widely accepted. Similarly, reproducibility of all immunohistochemical interpretations in pathology poses some limitations; however, reproducibility in the current series was excellent for both antibodies. Another limitation is the various histological cancer types but, at the same time, ours was a large series, allowing for comparison between them.

To conclude, this is the first large study comparing the immune microenvironment of various Bmets and their paired primaries. We have shown that PD-L1 expression of Bmets is concordant to that of the primary tumor in $75 \%$ of cases, with no factors being able to predict the discordance. CD8 expression is similarly concordant in most cases, with longer time to metastasis being the only factor predictive of discordance. Brain metastasis PD-L1 expression is dependent on primary tumor type, primary tumor PD-L1 expression, and brain metastasis CD8 infiltration. 
Brain metastasis CD8 infiltration depends on primary tumor type and primary tumor CD8 expression. Prognosis of Bmets is not associated with Bmets PD-L1 or CD8 expressions, while time to Bmets development is associated with the primary tumor type. Our findings show that Bmets may not be an immunoprivileged compartment, and studies exploring the efficacy of immunotherapy for this patient group could prove useful.

\section{Author affiliations}

${ }^{1}$ Pathology, University Hospital of Saint-Etienne, Saint-Etienne, France

${ }^{2}$ Radiotherapy, Lucien Neuwirth Cancer Institute, Saint-Etienne, France

${ }^{3}$ Dermatology, University Hospital of Saint-Etienne, Saint-Etienne, France

${ }^{4}$ Neurosurgery, University Hospital of Saint-Eteinne, Saint-Etienne, France

${ }^{5}$ Faculty of Medicine, Jazan University, Jazan, Saudi Arabia

${ }^{6}$ Pneumology and Thoracic Oncology, University Hospital of Saint-Etienne, SaintEtienne, France

Acknowledgements The authors would like to thank Mr Philippe Cosmo from the Tumorothèque/Centre de Ressources Biologiques de CHU Saint-Etienne (BRIF no. BB-0033-00041) for his assistance, as well as Mrs Josiane Beny and Mrs Carole Robert, and Mr Denis Chambouvet for their excellent technical assistance.

Contributors GK conceived the idea; GK, FC, and MP designed the study; MF performed the statistical analysis; FC and GK drafted the manuscript; and all authors corrected and approved the manuscript, and participated in data collection, analysis, and interpretation.

Funding The authors have not declared a specific grant for this research from any funding agency in the public, commercial or not-for-profit sectors.

Competing interests No, there are no competing interests.

Patient consent for publication Not required.

Ethics approval The study was approved by the institutional review board and local ethics committee with waivers of informed written consent given the retrospective nature of the study and the anonymization of all data (IRBN502017/ CHUSTE).

Provenance and peer review Not commissioned; externally peer reviewed. Data availability statement Data are available upon reasonable request.

Open access This is an open access article distributed in accordance with the Creative Commons Attribution Non Commercial (CC BY-NC 4.0) license, which permits others to distribute, remix, adapt, build upon this work non-commercially, and license their derivative works on different terms, provided the original work is properly cited, appropriate credit is given, any changes made indicated, and the use is non-commercial. See http://creativecommons.org/licenses/by-nc/4.0/.

\section{ORCID iDs}

Georgia Karpathiou http://orcid.org/0000-0003-0864-935X

Fabien Forest http://orcid.org/0000-0001-7582-3923

\section{REFERENCES}

1 Arvold ND, Lee EQ, Mehta MP, et al. Updates in the management of brain metastases. Neuro Oncol 2016;18:1043-65.

2 Nayak L, Lee EQ, Wen PY. Epidemiology of brain metastases. Curr Oncol Rep 2012;14:48-54.

3 Takei H, Rouah E, Ishida Y. Brain metastasis: clinical characteristics, pathological findings and molecular subtyping for therapeutic implications. Brain Tumor Pathol 2016;33:1-12.

4 Caponnetto S, Draghi A, Borch TH, et al. Cancer immunotherapy in patients with brain metastases. Cancer Immunol Immunother 2018;67:703-11.

5 Camidge DR, Lee EQ, Lin NU, et al. Clinical trial design for systemic agents in patients with brain metastases from solid tumours: a guideline by the response assessment in neuro-oncology brain metastases Working group. Lancet Oncol 2018;19:e20-32.

6 Goldberg SB, Gettinger SN, Mahajan A, et al. Pembrolizumab for patients with melanoma or non-small-cell lung cancer and untreated brain metastases: early analysis of a non-randomised, open-label, phase 2 trial. Lancet Oncol 2016;17:976-83.

7 Berghoff AS, Fuchs E, Ricken G, et al. Density of tumor-infiltrating lymphocytes correlates with extent of brain edema and overall survival time in patients with brain metastases. Oncoimmunology 2016;5:e1057388.

8 Berghoff AS, Ricken G, Widhalm G, et al. Tumour-Infiltrating lymphocytes and expression of programmed death ligand 1 (PD-L1) in melanoma brain metastases. Histopathology 2015;66:289-99.

9 Karpathiou G, Casteillo F, Giroult J-B, et al. Prognostic impact of immune microenvironment in laryngeal and pharyngeal squamous cell carcinoma: immune cell subtypes, immuno-suppressive pathways and clinicopathologic characteristics. Oncotarget 2017;8:19310-22.

10 Forest F, Yvorel V, Karpathiou G, et al. Histomolecular profiling of pleomorphic, spindle cell, and giant cell carcinoma of the lung for targeted therapies. Hum Pathol 2016;49:99-106.

11 Forest F, Stachowicz M-L, Casteillo F, et al. Egfr, KRAS, BRAF and HER2 testing in metastatic lung adenocarcinoma: value of testing on samples with poor specimen adequacy and analysis of discrepancies. Exp Mol Pathol 2017;103:306-10.

12 Casteillo F, Guy J-B, Dal-Col P, et al. Pathologic subtypes of lung adenocarcinoma brain metastasis is a strong predictor of survival after resection. Am J Surg Pathol 2018;42:1701-7.

13 Blank CU, Haanen JB, Ribas A, et al. The "cancer immunogram". Science 2016;352:658-60.

14 Duchnowska R, Pęksa R, Radecka B, et al. Immune response in breast cancer brain metastases and their microenvironment: the role of the PD-1/PD-L axis. Breast Cancer Res 2016;18:43.

15 Harter PN, Bernatz S, Scholz A, et al. Distribution and prognostic relevance of tumor-infiltrating lymphocytes (TILs) and PD-1/PDL1 immune checkpoints in human brain metastases. Oncotarget 2015;6:40836-49.

16 Mansfield AS, Aubry MC, Moser JC, et al. Temporal and spatial discordance of programmed cell death-ligand 1 expression and lymphocyte tumor infiltration between paired primary lesions and brain metastases in lung cancer. Ann Oncol 2016;27:1953-8.

17 Zhou J, Gong Z, Jia Q, et al. Programmed death ligand 1 expression and $\mathrm{CD}^{+}$tumor-infiltrating lymphocyte density differences between paired primary and brain metastatic lesions in non-small cell lung cancer. Biochem Biophys Res Commun 2018;498:751-7. 